\author{
A.S. Berdyshev ${ }^{1,2}$, A.R. Ryskan ${ }^{*}$ \\ ${ }^{1}$ Abai Kazakh National Pedagogical University, Almaty, Kazakhstan; \\ ${ }^{2}$ Institute of Information and Computational Technologies, Almaty, Kazakhstan \\ (E-mail: berdyshev@mail.ru,ryskan.a727@gmail.com)
}

\title{
Boundary value problem for the four-dimensional Gellerstedt equation
}

\begin{abstract}
In this work, the solvability of the problem with Neumann and Dirichlet boundary conditions for the Gellerstedt equation in four variables was investigated. The energy integral method was used to prove the uniqueness of the solution to the problem. In addition to it, formulas for differentiation, autotransformation, and decomposition of hypergeometric functions were applied. The solution was obtained explicitly and expressed by Lauricella's hypergeometric function.
\end{abstract}

Keywords: Gellerstedt equation, boundary value problem with mixed conditions, fundamental solution,

\section{Introduction}

The study of boundary value problems for degenerate equations is one of the important directions of modern theory of partial differential equations. The solution of many boundary value problems for partial differential equations has an applied nature [1-2]. The boundary value problems for degenerate elliptic equations were well studied in works $[3-6]$.

In the formulation of problems and questions of the solvability of local and nonlocal boundary value problems for degenerate elliptic equations, fundamental solutions of these equations are essentially used [7]. The explicit form of the fundamental solutions makes it possible to correctly formulate the problem statement and study in detail the various properties of the considered equation solutions.

Fundamental solutions of degenerate elliptic equations are expressed in terms of the Lauricella's multidimensional hypergeometric functions and the Gauss hypergeometric function of one variable. Many problems of natural science, such as problems of dynamics and heat conduction, the theory of electromagnetic oscillations, aerodynamics, quantum mechanics, quantum chemistry, potential theory, etc. lead to the study of various properties of multidimensional hypergeometric functions [8-14].

For two-dimensional and three-dimensional elliptic equations with singular coefficients, fundamental solutions were constructed, which were applied in the study of the various problems solvability in many works [15-19].

In [20] fundamental solutions for the generalized Gellerstedt equation of four variables were constructed

$$
y^{m} z^{k} t^{l} u_{x x}+x^{n} z^{k} t^{l} u_{y y}+x^{n} y^{m} t^{l} u_{z z}+x^{n} y^{m} z^{k} u_{t t}=0, m, n, k, l>0, m, n, k, l \equiv \text { const. }
$$

Since the generalized Gellerstedt equation has four hypersurfaces of degeneration of the equation type, accordingly sixteen fundamental solutions were obtained. It was proved that the fundamental solutions have a singularity of the order $\frac{1}{r^{2}}$, at $r \rightarrow 0$, where $r=\sqrt{x^{2}+y^{2}+z^{2}+t^{2}}$.

These fundamental solutions are expressed in terms of Lauricella's hypergeometric functions, each of the fundamental solutions is applied in solving the corresponding boundary value problems [21-23].

\section{Preliminary information}

By definition, the Gauss hypergeometric function has the form

\footnotetext{
*Corresponding author.

E-mail: ryskan.a727@gmail.com
} 


$$
F(a, b ; c ; x)=\sum_{n=0}^{\infty} \frac{(a)_{n}(b)_{n}}{(c)_{n} n !} x^{n}, \quad c \neq 0,-1,-2, \ldots,
$$

where

$$
(a)_{n}=a(a+1)(a+2) \ldots(a+n-1)=\frac{\Gamma(a+n)}{\Gamma(a)}(n=0,1,2,3, \ldots)
$$

is Pochhammer symbol. Here $\Gamma(a)$ is Euler's gamma function, for it the formula of the doubled argument is valid $[24 ; 19,(15)]$

$$
\Gamma(2 z)=2^{2 z-1} \pi^{-\frac{1}{2}} \Gamma(z) \Gamma\left(z+\frac{1}{2}\right) .
$$

We present the main properties of the Euler's gamma function, the Gauss hypergeometric function, and Lauricella's hypergeometric function of many variables, which will be used in what follows.

The Gauss hypergeometric function has the following property $[25 ; 3,(5)]$ :

$$
F(a, b ; c ; 1)=\frac{\Gamma(c) \Gamma(c-a-b)}{\Gamma(c-a) \Gamma(c-b)}, \quad \operatorname{Re}(c-a-b)>0 .
$$

The Gauss hypergeometric function satisfies the Bolz autotransformation formula [26; 64, (22)]:

$$
F(a, b ; c ; x)=(1-x)^{-b} F\left(c-a, b ; c ; \frac{x}{x-1}\right) .
$$

Lauricella's hypergeometric function of $n$ variables $[25 ; 114]$

$$
\begin{aligned}
& F_{D}^{(n)}\left(a ; b_{1}, b_{2}, b_{3}, \ldots, b_{n} ; c ; x_{1}, x_{2}, \ldots, x_{n}\right)=\sum_{m_{1}, \ldots, m_{n}=0}^{\infty} \frac{(a)_{m_{1}+\ldots+m_{n}}\left(b_{1}\right)_{m_{1}} \ldots\left(b_{n}\right)_{m_{n}}}{(c)_{m_{1}+\ldots+m_{n}} m_{1} ! \ldots m_{n} !} x_{1}^{m_{1}} \ldots x_{n}^{m_{n}}, \\
& \left(\left|x_{1}\right|<1,\left|x_{2}\right|<1, \ldots,\left|x_{n}\right|<1\right),
\end{aligned}
$$

the form $[25 ; 117]$

$$
\begin{aligned}
& F_{D}^{(n)}\left(a ; b_{1}, b_{2}, b_{3}, \ldots, b_{n} ; c ; 1,1, \ldots, 1\right)=\frac{\Gamma(c) \Gamma\left(c-a-b_{1}-b_{2}-\ldots-b_{n}\right)}{\Gamma(c-a) \Gamma\left(c-b_{1}-b_{2}-\ldots-b_{n}\right)}, n=1,2, \ldots \\
& \operatorname{Re}\left(c-a-b_{1}-b_{2}-\ldots-b_{n}\right)>0, \quad c \neq 0,-1,-2, \ldots
\end{aligned}
$$

in the case when all variables in (4) take the value 1.

Lauricella's hypergeometric function in the case of four variables has the form $[25 ; 114,(1)]$ :

$$
\begin{aligned}
& F_{A}^{(4)}\left(a ; b_{1}, b_{2}, b_{3}, b_{4} ; c_{1}, c_{2}, c_{3}, c_{4} ; x, y, z, t\right)=\sum_{m, n, p, q}^{\infty} \frac{(a)_{m+n+p+q}\left(b_{1}\right)_{m}\left(b_{2}\right)_{n}\left(b_{3}\right)_{p}\left(b_{4}\right)_{q}}{\left(c_{1}\right)_{m}\left(c_{2}\right)_{n}\left(c_{3}\right)_{p}\left(c_{4}\right)_{q} m ! n ! p ! q !} x^{m} y^{n} z^{p} t^{q}, \\
& (|x|+|y|+|z|+|t|<1) .
\end{aligned}
$$
[27]:

The validity of the decomposition formula for a hypergeometric function of three variables, was proved in

$$
\begin{aligned}
& F_{A}^{(3)}\left(a, b_{1}, b_{2}, b_{3} ; c_{1}, c_{2}, c_{3} ; x, y, z\right)=\sum_{n_{1}, n_{2}, n_{3}=0}^{\infty} \frac{(a)_{n_{1}+n_{2}+n_{3}}\left(b_{1}\right)_{n_{1}+n_{2}}\left(b_{2}\right)_{n_{1}+n_{3}}\left(b_{3}\right)_{n_{2}+n_{3}}}{\left(c_{1}\right)_{n_{1}+n_{2}}\left(c_{2}\right)_{n_{1}+n_{3}}\left(c_{3}\right)_{n_{2}+n_{3}} n_{1} ! n_{2} ! n_{3} !} \times \\
& \times x^{n_{1}+n_{2}} y^{n_{1}+n_{3}} z^{n_{2}+n_{3}} F\left(a+n_{1}+n_{2}, b_{1}+n_{1}+n_{2} ; c_{1}+n_{1}+n_{2} ; x\right) \\
& \times F\left(a+n_{1}+n_{2}+n_{3}, b_{2}+n_{1}+n_{3} ; c_{2}+n_{1}+n_{3} ; y\right) \\
& \times F\left(a+n_{1}+n_{2}+n_{3}, b_{3}+n_{2}+n_{3} ; c_{3}+n_{2}+n_{3} ; z\right) .
\end{aligned}
$$

We also use the formula for the differentiation of hypergeometric functions of three variables [25] 


$$
\begin{aligned}
& \frac{\partial^{i+j+k}}{\partial x^{i} \partial y^{j} \partial z^{k}} F_{A}^{(3)}\left(\alpha ; \beta_{1}, \beta_{2}, \beta_{3} ; \gamma_{1}, \gamma_{2}, \gamma_{3} ; x, y, z\right)=\frac{(\alpha)_{i+j+k}\left(\beta_{1}\right)_{i}\left(\beta_{2}\right)_{j}\left(\beta_{3}\right)_{k}}{\left(\gamma_{1}\right)_{i}\left(\gamma_{2}\right)_{j}\left(\gamma_{3}\right)_{k}} \times \\
& \times F_{A}^{(3)}\left(\alpha+i+j+k ; \beta_{1}+i, \beta_{2}+j, \beta_{3}+k ; \gamma_{1}+i, \gamma_{2}+j, \gamma_{3}+k ; x, y, z\right), \\
& i, j, k \in \mathbb{N}_{0}=\{0,1,2, \ldots\} .
\end{aligned}
$$

For Lauricella's function $F_{A}^{(n)}$ the following adjacent relations are valid

$$
\begin{aligned}
& \frac{b_{1}}{c_{1}} x_{1} F_{A}\left(a+1 ; b_{1}+1, b_{2}, \ldots, b_{n} ; c_{1}+1, c_{2}, \ldots, c_{n} ; x_{1}, \ldots, x_{n}\right) \\
& +\frac{b_{2}}{c_{2}} x_{2} F_{A}\left(a+1 ; b_{1}, b_{2}+1, \ldots, b_{n} ; c_{1}, c_{2}+1, \ldots, c_{n} ; x_{1}, \ldots, x_{n}\right) \\
& +\ldots+\frac{b_{n}}{c_{n}} x_{n} F_{A}\left(a+1 ; b_{1}, b_{2}, \ldots, b_{n}+1 ; c_{1}, c_{2}, \ldots, c_{n}+1 ; x_{1}, \ldots, x_{n}\right)= \\
& =F_{A}\left(a+1 ; b_{1}, \ldots, b_{n} ; c_{1}, \ldots, c_{n} ; x_{1}, \ldots, x_{n}\right)-F_{A}\left(a ; b_{1}, \ldots, b_{n} ; c_{1}, \ldots, c_{n} ; x_{1}, \ldots, x_{n}\right) .
\end{aligned}
$$

To calculate the value of a multiple integral, we use the formula $[28 ; 637,(3)]$

$$
\begin{gathered}
\int_{0}^{\infty} \int_{0}^{\infty} \ldots \int_{0}^{\infty} \frac{x_{1}^{p_{1}-1} x_{2}^{p_{2}-1} \ldots x_{n}^{p_{n}-1}}{\left[1+\left(r_{1} x_{1}\right)^{q_{1}}+\left(r_{2} x_{2}\right)^{q_{2}}+\ldots+\left(r_{n} x_{n}\right)^{q_{n}}\right]^{s}} d x_{1} d x_{2} \ldots d x_{n}= \\
=\frac{\Gamma\left(\frac{p_{1}}{q_{1}}\right) \Gamma\left(\frac{p_{2}}{q_{2}}\right) \ldots \Gamma\left(\frac{p_{n}}{q_{n}}\right)}{q_{1} q_{2} \ldots q_{n} r_{1}{ }^{p_{1} q_{1}} r_{2} p_{2} q_{2} \ldots r_{n} p_{n} q_{n}} \frac{\Gamma\left(s-\frac{p_{1}}{q_{1}}-\frac{p_{2}}{q_{2}}-\ldots-\frac{p_{n}}{q_{n}}\right)}{\Gamma(s)},\left(p_{i}>0, q_{i}>0, r_{i}>0, s>0\right),
\end{gathered}
$$

and for integrals expressed in terms of the beta function, the formulas [24; 25, (16), (19)]

$$
\begin{gathered}
\int_{0}^{\infty}\left(1+b t^{z}\right)^{-y} t^{x} d t=z^{-1} b^{-\frac{x+1}{z}} \beta\left(\frac{x+1}{z}, y-\frac{x+1}{z}\right),\left(z>0, b>0,0<\operatorname{Re} \frac{x+1}{z}<\operatorname{Re} y\right) \\
\int_{0}^{\frac{\pi}{2}}(\sin t)^{2 x-1}(\cos t)^{2 y-1} d t=\frac{1}{2} \beta(x, y),(\operatorname{Re} x>0, \operatorname{Re} y>0)
\end{gathered}
$$

\section{Statement of the problem}

Considering the generalized Gellerstedt equation:

$$
H(u)=y^{m} z^{k} t^{l} u_{x x}+x^{n} z^{k} t^{l} u_{y y}+x^{n} y^{m} t^{l} u_{z z}+x^{n} y^{m} z^{k} u_{t t}=0, m, n, k, l>0, m, n, k, l \equiv \text { const },
$$

we introduce the following notations:

$$
\begin{aligned}
& D=\{(x, y, z, t): x>0, y>0, z>0, t>0\}, \\
& S_{1}=\{(0, y, z, t): x=0, y>0, z>0, t>0\}, \\
& S_{2}=\{(x, 0, z, t): x>0, y=0, z>0, t>0\}, \\
& S_{3}=\{(x, y, 0, t): x>0, y>0, z=0, t>0\}, \\
& S_{4}=\{(x, y, z, 0): x>0, y>0, z>0, t=0\}, \\
& R^{2}=\frac{4}{(n+2)^{2}} x^{n+2}+\frac{4}{(m+2)^{2}} y^{m+2}+\frac{4}{(k+2)^{2}} z^{k+2}+\frac{4}{(l+2)^{2}} t^{l+2} .
\end{aligned}
$$

Problem $N D_{2}$. Find a regular solution $u(x, y, z, t)$ of the equation (13) from the class $C(\bar{D}) \cap C^{1}\left(D \cup \overline{S_{3}} \cup \overline{S_{4}}\right) \cap$ $C^{2}(D)$ satisfying the condition: 


$$
\begin{aligned}
&\left.u(x, y, z, t)\right|_{x=0}=\tau_{1}(y, z, t), \quad(y, z, t) \in \overline{S_{1}}, \\
&\left.u(x, y, z, t)\right|_{y=0}=\tau_{2}(x, z, t), \quad(x, z, t) \in \overline{S_{2}}, \\
&\left.\frac{\partial}{\partial z} u(x, y, z, t)\right|_{z=0}=\nu_{3}(x, y, t), \quad(x, y, t) \in S_{3}, \\
&\left.\frac{\partial}{\partial t} u(x, y, z, t)\right|_{t=0}=\nu_{4}(x, y, z), \quad(x, y, z) \in S_{4}, \\
& \lim _{R \rightarrow \infty} u(x, y, z, t)=0,
\end{aligned}
$$

where $\tau_{1}(y, z, t), \tau_{2}(x, z, t), \nu_{3}(x, y, t), \nu_{4}(x, y, z) \in \mathbb{C}$ are given continuous functions, moreover the function $\nu_{3}(x, y, t), \nu_{4}(x, y, z)$ at the origin of coordinates can go to integrable order infinity. Also, for the large enough values $R$, the following inequalities hold:

$$
\begin{gathered}
\left|\tau_{1}(y, z, t)\right| \leq \frac{c_{1}}{\left[1+\frac{4}{(n+2)^{2}} y^{m+2}+\frac{4}{(k+2)^{2}} z^{k+2}+\frac{4}{(l+2)^{2}} t^{l+2}\right]^{\varepsilon_{1}}}, \\
\left|\tau_{2}(x, z, t)\right| \leq \frac{c_{2}}{\left[1+\frac{4}{(n+2)^{2}} x^{n+2}+\frac{4}{(k+2)^{2}} z^{k+2}+\frac{4}{(l+2)^{2}} t^{l+2}\right]^{\varepsilon_{2}}}, \\
\left|\nu_{3}(x, y, t)\right| \leq \frac{c_{3}}{\left[1+\frac{4}{(n+2)^{2}} x^{n+2}+\frac{4}{(m+2)^{2}} y^{m+2}+\frac{4}{(l+2)^{2}} t^{l+2}\right]^{\frac{1-2 \gamma+\varepsilon_{3}}{2}}}, \\
\left|\nu_{4}(x, y, z)\right| \leq \frac{c_{4}}{\left[1+\frac{4}{(n+2)^{2}} x^{n+2}+\frac{4}{(m+2)^{2}} y^{m+2}+\frac{4}{(k+2)^{2}} z^{k+2}\right]^{\frac{1-2 \delta+\varepsilon_{4}}{2}}},
\end{gathered}
$$

here $c_{1}, c_{2}, c_{3}, c_{4}>0$ and $\varepsilon_{1}, \varepsilon_{2}, \varepsilon_{3}, \varepsilon_{4}$ are small enough positive numbers.

Theorem 1 . The boundary value problem $N D_{2}$ has at most one solution.

Proof. Let $u(x, y, z, t)$ be the solution of a homogeneous problem $N D_{2}$, i.e. $u(x, y, z, t)$ is the solution of the equation (13) satisfying the conditions (14)-(18).

By $D_{R}$ we denote the bounded domain with the boundary $\partial D_{R}=S_{1 R} \cup S_{2 R} \cup S_{3 R} \cup S_{4 R} \cup \sigma_{R}$, where $S_{1 R}=S_{1} \cap\{x=0,0<y<R, 0<z<R, 0<t<R\}, S_{2 R}=S_{2} \cap\{0<x<R, y=0,0<z<R, 0<t<R\}$, $S_{3 R}=S_{3} \cap\{0<x<R, 0<y<R, z=0,0<t<R\}, S_{4 R}=S_{4} \cap\{0<x<R, 0<y<R, 0<z<R, t=0\}$, $\sigma_{R}=\left\{(x, y, z, t): \frac{4}{(n+2)^{2}} x^{n+2}+\frac{4}{(m+2)^{2}} y^{m+2}+\frac{4}{(k+2)^{2}} z^{k+2}+\frac{4}{(l+2)^{2}} t^{l+2}=R^{2}, x \geq 0, y \geq 0, z \geq 0, t \geq 0\right\}$.

Choosing large enough $R$, we integrate equation (13) over the domain $D_{R}$, previously multiplied it by a function $u(x, y, z, t)$, we obtain

$$
\iiint \int_{D_{R}}\left[y^{m} z^{k} t^{l} u u_{x x}+x^{n} z^{k} t^{l} u u_{y y}+x^{n} y^{m} t^{l} u u_{z z}+x^{n} y^{m} z^{k} u u_{t t}\right] d x d y d z d t=0 .
$$

Taking into account (23) we obtain the following equalities:

$$
\begin{aligned}
& y^{m} z^{k} t^{l} u u_{x x}=\frac{\partial}{\partial x}\left(y^{m} z^{k} t^{l} u u_{x}\right)-y^{m} z^{k} t^{l} u_{x}^{2}, \quad x^{n} z^{k} t^{l} u u_{y y}=\frac{\partial}{\partial y}\left(x^{n} z^{k} t^{l} u u_{y}\right)-x^{n} z^{k} t^{l} u_{y}^{2}, \\
& x^{n} y^{m} t^{l} u u_{z z}=\frac{\partial}{\partial z}\left(x^{n} y^{m} t^{l} u u_{z}\right)-x^{n} y^{m} t^{l} u_{z}^{2}, \quad x^{n} y^{m} z^{k} u u_{t t}=\frac{\partial}{\partial t}\left(x^{n} y^{m} z^{k} u u_{t}\right)-x^{n} y^{m} z^{k} u_{t}^{2},
\end{aligned}
$$

after applying the Gauss-Ostrogradsky formula, we have

$$
\begin{aligned}
& \iiint \int_{D_{R}}\left[y^{m} z^{k} t^{l} u_{x}^{2}+x^{n} z^{k} t^{l} u_{y}^{2}+x^{n} y^{m} t^{l} u_{z}^{2}+x^{n} y^{m} z^{k} u_{t}^{2}\right] d x d y d z d t= \\
& =\iiint_{S_{1 R}} y^{m} z^{k} t^{l} \tau_{1} u_{x} d y d z d t+\iiint_{S_{2 R}} x^{n} z^{k} t^{l} \tau_{2} u_{y} d x d z d t+\iiint_{S_{3 R}} x^{n} y^{m} t^{l} u \nu_{3} d x d y d t \\
& +\iiint_{S_{4 R}} x^{n} y^{m} z^{k} u \nu_{4} d x d y d z+\iiint_{\sigma_{R}} x^{n} y^{m} z^{k} t^{l} u \frac{\partial u}{\partial n} d S
\end{aligned}
$$


where,

$$
\frac{\partial u}{\partial n}=u_{x} \cos (n, x)+u_{y} \cos (n, y)+u_{z} \cos (n, z)+u_{t} \cos (n, t),
$$

$\cos (n, x) d S=d y d z d t, \quad \cos (n, y) d S=d x d z d t, \quad \cos (n, z) d S=d x d y d t, \cos (n, t) d S=d x d y d z, n$ is outer normal to $\partial D_{R}$.

Since for the function $u \tau_{1}=\tau_{2}=\nu_{3}=\nu_{4}=0$, then from (24) we have

$$
\iiint \int_{D_{R}}\left[y^{m} z^{k} t^{l} u_{x}^{2}+x^{n} z^{k} t^{l} u_{y}^{2}+x^{n} y^{m} t^{l} u_{z}^{2}+x^{n} y^{m} z^{k} u_{t}^{2}\right] d x d y d z d t=\iiint_{\sigma_{R}} x^{n} y^{m} z^{k} t^{l} u \frac{\partial u}{\partial n} d S .
$$

By virtue of condition (18) for $R \rightarrow \infty \lim _{R \rightarrow \infty} \iiint_{\sigma_{R}} x^{n} y^{m} z^{k} t^{l} u \frac{\partial u}{\partial n} d S=0$, then from (25) we have

$$
\iiint \int_{D}\left[y^{m} z^{k} t^{l} u_{x}^{2}+x^{n} z^{k} t^{l} u_{y}^{2}+x^{n} y^{m} t^{l} u_{z}^{2}+x^{n} y^{m} z^{k} u_{t}^{2}\right] d x d y d z d t \equiv 0 .
$$

From (26), we get $u_{x}=u_{y}=u_{z}=u_{t}=0$, which means $u=$ const, and from the conditions $\left.u\right|_{x=0}=\left.u\right|_{y=0}=$ $\left.u_{z}\right|_{z=0}=\left.u_{t}\right|_{t=0}=0$ follows that $u \equiv 0$. So, we have proved the uniqueness of the problem $N D_{2}$.

\section{Existence of a problem solution}

The solution to the $N D_{2}$ problem has the form

$$
\begin{aligned}
u\left(x_{0}, y_{0}, z_{0}, t_{0}\right) & =\left.\int_{0}^{\infty} \int_{0}^{\infty} \int_{0}^{\infty} y^{m} z^{k} t^{l} \tau_{1}(y, z, t) \frac{\partial}{\partial x} g_{6}\left(x, y, z, t ; x_{0}, y_{0}, z_{0}, t_{0}\right)\right|_{x=0} d y d z d t+ \\
& +\left.\int_{0}^{\infty} \int_{0}^{\infty} \int_{0}^{\infty} x^{n} z^{k} t^{l} \tau_{2}(x, z, t) \frac{\partial}{\partial y} g_{6}\left(x, y, z, t ; x_{0}, y_{0}, z_{0}, t_{0}\right)\right|_{y=0} d x d z d t- \\
& -\int_{0}^{\infty} \int_{0}^{\infty} \int_{0}^{\infty} x^{n} y^{m} t^{l} \nu_{3}(x, y, t) g_{6}\left(x, y, 0, t ; x_{0}, y_{0}, z_{0}, t_{0}\right) d x d y d t- \\
& -\int_{0}^{\infty} \int_{0}^{\infty} \int_{0}^{\infty} x^{n} y^{m} z^{k} \nu_{4}(x, y, z) g_{6}\left(x, y, z, 0 ; x_{0}, y_{0}, z_{0}, t_{0}\right) d x d y d z
\end{aligned}
$$

where

$$
\begin{aligned}
& g_{6}\left(x, y, z, t ; x_{0}, y_{0}, z_{0}, t_{0}\right)=k_{6}\left(\frac{4}{n+2}\right)^{\frac{4}{n+2}}\left(\frac{4}{m+2}\right)^{\frac{4}{m+2}}\left(r^{2}\right)^{\alpha+\beta-\gamma-\delta-3} x y x_{0} y_{0} \times \\
& \times F_{A}^{(4)}(3-\alpha-\beta+\gamma+\delta ; 1-\alpha, 1-\beta, \gamma, \delta ; 2-2 \alpha, 2-2 \beta, 2 \gamma, 2 \delta ; \xi, \eta, \zeta, \varsigma)
\end{aligned}
$$

is fundamental solution to the equation (13). Here function $F_{A}^{(4)}$ is Lauricella's function (6),

$$
\begin{aligned}
k_{6}=\frac{1}{4 \pi^{2}}\left(\frac{4}{n+2}\right)^{2 \alpha}\left(\frac{4}{m+2}\right)^{2 \beta}\left(\frac{4}{k+2}\right)^{2 \gamma}\left(\frac{4}{l+2}\right)^{2 \delta} \times \\
\times \frac{\Gamma(3-\alpha-\beta+\gamma+\delta) \Gamma(1-\alpha) \Gamma(1-\beta) \Gamma(\gamma) \Gamma(\delta)}{\Gamma(2-2 \alpha) \Gamma(2-2 \beta) \Gamma(2 \gamma) \Gamma(2 \delta)}, \\
\xi=\frac{r^{2}-r_{1}^{2}}{r^{2}}, \quad \eta=\frac{r^{2}-r_{2}^{2}}{r^{2}}, \quad \zeta=\frac{r^{2}-r_{3}^{2}}{r^{2}}, \quad \varsigma=\frac{r^{2}-r_{4}^{2}}{r^{2}}
\end{aligned}
$$

Mathematics series. № 4(104)/2021 


$$
\begin{aligned}
& \left.\begin{array}{r}
r^{2} \\
r_{1}^{2} \\
r_{2}^{2} \\
r_{3}^{2} \\
r_{4}^{2}
\end{array}\right\}=\left(\begin{array}{rl}
- \\
\frac{2}{n+2} x^{\frac{n+2}{2}} & -\frac{2}{n+2} x_{0}^{\frac{n+2}{2}} \\
& -
\end{array}\right)^{2}+\left(\begin{array}{c} 
\\
\frac{2}{m+2} y^{\frac{m+2}{2}} \\
- \\
- \\
-
\end{array}\right)^{2} \\
& +\left(\begin{array}{rl}
\frac{-}{k+2} z^{\frac{k+2}{2}} & -\frac{2}{k+2} z_{0}^{\frac{k+2}{2}} \\
& -
\end{array}\right)^{2}+\left(\begin{array}{rl}
2 & - \\
\frac{2}{l+2} t^{\frac{l+2}{2}} & -\frac{2}{l+2} t_{0}^{\frac{l+2}{2}} \\
& +
\end{array}\right)^{2} \\
& \alpha=\frac{n}{2(n+2)}, \quad \beta=\frac{m}{2(m+2)}, \quad \gamma=\frac{k}{2(k+2)}, \quad \delta=\frac{l}{2(l+2)} .
\end{aligned}
$$

Since the function $q_{6}$ is a fundamental solution to equation (13), it is obvious that the solution to problem (27) satisfies equation (13).

Let us prove that function (27) satisfies conditions (14) - (17) of problem $N D_{2}$. We apply differentiation formulas (8) and decomposition of hypergeometric functions (9) to (27) and represent (27) as the sum:

$$
u\left(x_{0}, y_{0}, z_{0}, t_{0}\right)=I_{1}\left(x_{0}, y_{0}, z_{0}, t_{0}\right)+I_{2}\left(x_{0}, y_{0}, z_{0}, t_{0}\right)+I_{3}\left(x_{0}, y_{0}, z_{0}, t_{0}\right)+I_{4}\left(x_{0}, y_{0}, z_{0}, t_{0}\right),
$$

where

$$
\begin{gathered}
I_{1}\left(x_{0}, y_{0}, z_{0}, t_{0}\right)=k_{6}\left(\frac{4}{n+2}\right)^{\frac{4}{n+2}}\left(\frac{4}{m+2}\right)^{\frac{4}{m+2}} x_{0} y_{0} \int_{0}^{\infty} \int_{0}^{\infty} \int_{0}^{\infty} y^{m+1} z^{k} t^{l} \tau_{1}(y, z, t) \times \\
\times\left.\left(r^{2}\right)^{\alpha+\beta-\gamma-\delta-3} F_{A}^{(3)}(3-\alpha-\beta+\gamma+\delta ; 1-\beta, \gamma, \delta ; 2-2 \beta, 2 \gamma, 2 \delta ; \eta, \zeta, \varsigma)\right|_{x=0} d y d z d t, \\
I_{2}\left(x_{0}, y_{0}, z_{0}, t_{0}\right)=k_{6}\left(\frac{4}{n+2}\right)^{\frac{4}{n+2}}\left(\frac{4}{m+2}\right)^{\frac{4}{m+2}} x_{0} y_{0} \int_{0}^{\infty} \int_{0}^{\infty} \int_{0}^{\infty} x^{n+1} z^{k} t^{l} \tau_{2}(x, z, t) \times \\
\times\left.\left(r^{2}\right)^{\alpha+\beta-\gamma-\delta-3} F_{A}^{(3)}(3-\alpha-\beta+\gamma+\delta ; 1-\alpha, \gamma, \delta ; 2-2 \alpha, 2 \gamma, 2 \delta ; \xi, \zeta, \varsigma)\right|_{y=0} d x d z d t, \\
I_{3}\left(x_{0}, y_{0}, z_{0}, t_{0}\right)=-k_{6}\left(\frac{4}{n+2}\right)^{\frac{4}{n+2}}\left(\frac{4}{m+2}\right)^{\frac{4}{m+2}} x_{0} y_{0} \int_{0}^{\infty} \int_{0}^{\infty} \int_{0}^{\infty} x^{n+1} y^{m+1} t^{l} \nu_{3}(x, y, t) \times \\
\times\left.\left(r^{2}\right)^{\alpha+\beta-\gamma-\delta-3} F_{A}^{(3)}(3-\alpha-\beta+\gamma+\delta ; 1-\alpha, 1-\beta, \delta ; 2-2 \alpha, 2-2 \beta, 2 \delta ; \xi, \eta, \varsigma)\right|_{z=0} d x d y d t, \\
I_{4}\left(x_{0}, y_{0}, z_{0}, t_{0}\right)=-k_{6}\left(\frac{4}{n+2}\right)^{\frac{4}{n+2}}\left(\frac{4}{m+2}\right)^{\frac{4}{m+2}} x_{0} y_{0} \int_{0}^{\infty} \int_{0}^{\infty} \int_{0}^{\infty} x^{n+1} y^{m+1} z^{k} \nu_{4}(x, y, z) \times \\
\left.\left(r^{2}\right)^{\alpha+\beta-\gamma-\delta-3} F_{A}^{(3)}(3-\alpha-\beta+\gamma+\delta ; 1-\alpha, 1-\beta, \gamma ; 2-2 \alpha, 2-2 \beta, 2 \gamma ; \xi, \eta, \zeta)\right|_{t=0} d x d y d z .
\end{gathered}
$$

Let us check condition (14). Consider the first term of the solution, written in the form (29), function (30). We decompose the function $F_{A}^{(3)}$ in (30) by formula (7), then after performing some transformations in (30) and applying the Bolz autotransformation formula (3), we obtain

$$
\begin{aligned}
& F_{A}(3-\alpha-\beta+\gamma+\delta ; 1-\beta, \gamma, \delta ; 2-2 \beta, 2 \gamma, 2 \delta ; \eta, \zeta, \varsigma)= \\
& \quad=\left(r^{2}\right)^{1-\beta+\gamma+\delta}\left(r_{2}^{2}\right)^{\beta-1}\left(r_{3}^{2}\right)^{-\gamma}\left(r_{4}^{2}\right)^{-\delta} P_{1}\left(0, y, z, t ; x_{0}, y_{0}, z_{0}, t_{0}\right),
\end{aligned}
$$


where

$$
\begin{aligned}
& P_{1}\left(0, y, z, t ; x_{0}, y_{0}, z_{0}, t_{0}\right)=\sum_{l_{1}, l_{2}, l_{3}=0}^{\infty} \frac{(3-\alpha-\beta+\gamma+\delta)_{l_{1}+l_{2}+l_{3}}(1-\beta)_{l_{1}+l_{2}}(\gamma)_{l_{1}+l_{3}}(\delta)_{l_{2}+l_{3}}}{(2-2 \beta)_{l_{1}+l_{2}}(2 \gamma)_{l_{1}+l_{3}}(2 \delta)_{l_{2}+l_{3}} l_{1} ! l_{2} ! l_{3} !} \\
& \quad \times\left(\frac{r_{2}^{2}-r^{2}}{r_{2}^{2}}\right)^{l_{1}+l_{2}}\left(\frac{r_{3}^{2}-r^{2}}{r_{3}^{2}}\right)^{l_{1}+l_{3}}\left(\frac{r_{4}^{2}-r^{2}}{r_{4}^{2}}\right)^{l_{2}+l_{3}} \\
& \quad \times F\left(\alpha-\beta-\gamma-\delta-1,1-\beta+l_{1}+l_{2} ; 2-2 \beta+l_{1}+l_{2} ; \frac{r_{2}^{2}-r^{2}}{r_{2}^{2}}\right) \\
& \quad \times F\left(\alpha+\beta+\gamma-\delta-3-l_{2}, \gamma+l_{1}+l_{3} ; 2 \gamma+l_{1}+l_{3} ; \frac{r_{3}^{2}-r^{2}}{r_{3}^{2}}\right) \\
& \quad \times F\left(\alpha+\beta-\gamma+\delta-3-l_{1}, \delta+l_{2}+l_{3} ; 2 \delta+l_{2}+l_{3} ; \frac{r_{4}^{2}-r^{2}}{r_{4}^{2}}\right) .
\end{aligned}
$$

Thus, substituting (34) into (30), we have

$$
\begin{aligned}
& I_{1}\left(x_{0}, y_{0}, z_{0}, t_{0}\right)=k_{6}\left(\frac{4}{n+2}\right)^{\frac{4}{n+2}}\left(\frac{4}{m+2}\right)^{\frac{4}{m+2}} x_{0} y_{0} \times \\
& \times\left.\int_{0}^{\infty} \int_{0}^{\infty} \int_{0}^{\infty} y^{m+1} z^{k} t^{l} \tau_{1}(y, z, t) \frac{P_{1}\left(0, y, z, t ; x_{0}, y_{0}, z_{0}, t_{0}\right)}{\left(r^{2}\right)^{2-\alpha}\left(r_{2}^{2}\right)^{1-\beta}\left(r_{3}^{2}\right)^{\gamma}\left(r_{4}^{2}\right)^{\delta}}\right|_{x=0} d y d z d t .
\end{aligned}
$$

In (36), we make the change of variables

$$
\begin{gathered}
\frac{2}{m+2} y^{\frac{m+2}{2}}= \\
\frac{2}{m+2} y_{0}^{\frac{m+2}{2}}+\frac{2}{n+2} x_{0}^{\frac{n+2}{2}} s_{1}, \frac{2}{k+2} z^{\frac{k+2}{2}}=\frac{2}{k+2} z_{0}^{\frac{k+2}{2}}+\frac{2}{n+2} x_{0}^{\frac{n+2}{2}} s_{2} \\
\frac{2}{l+2} t^{\frac{l+2}{2}}=\frac{2}{l+2} t_{0}^{\frac{l+2}{2}}+\frac{2}{n+2} x_{0}^{\frac{n+2}{2}} s_{3} .
\end{gathered}
$$

Then, we obtain the following equality

$$
\begin{aligned}
& I_{1}\left(x_{0}, y_{0}, z_{0}, t_{0}\right)=k_{6}\left(\frac{4}{n+2}\right)^{\frac{4}{n+2}}\left(\frac{4}{m+2}\right)^{\frac{4}{m+2}} x_{0} y_{0}\left(\frac{2}{n+2} x_{0}^{\frac{n+2}{2}}\right)^{3} \\
& \int_{-a}^{\infty} \int_{-b}^{\infty} \int_{-c}^{\infty}\left[\left[\frac{m+2}{2}\left(\frac{2}{m+2} y_{0}^{\frac{m+2}{2}}+\frac{2}{n+2} x_{0}^{\frac{n+2}{2}} s_{1}\right)\right]^{\frac{2}{m+2}}\right]^{m+1}\left[\left[\frac{k+2}{2}\left(\frac{2}{k+2} z_{0}^{\frac{k+2}{2}}+\frac{2}{n+2} x_{0}^{\frac{n+2}{2}} s_{2}\right)\right]^{\frac{2}{k+2}}\right]^{k} \\
& \left.\times\left.\left[\left[\frac{l+2}{2}\left(\frac{2}{l+2} t_{0}^{\frac{l+2}{2}}+\frac{2}{n+2} x_{0}^{\frac{n+2}{2}} s_{3}\right)\right]^{\frac{2}{l+2}}\right]^{l} \frac{P_{1}\left(0, y, z, t ; x_{0}, y_{0}, z_{0}, t_{0}\right)}{\left(1+s_{1}^{2}+s_{2}^{2}+s_{3}^{2}\right)^{2-\alpha}\left(r_{2}^{2}\right)^{1-\beta}\left(r_{3}^{2}\right)^{\gamma}\left(r_{4}^{2}\right)^{\delta}}\right|_{x=0}, \frac{2}{2}\left(\frac{2}{k+2} z_{0}^{\frac{k+2}{2}}+\frac{2}{n+2} x_{0}^{\frac{n+2}{2}} s_{2}\right)\right]^{\frac{2}{k+2}}, \\
& \times \tau_{1}\left(\left[\frac{m+2}{2}\left(\frac{2}{m+2} y_{0}^{\frac{m+2}{2}}+\frac{2}{n+2} x_{0}^{\frac{n+2}{2}} s_{1}\right)\right]^{\frac{2}{m+2}}\left[\frac{k+2}{2}\left(\frac{2}{l+2} t_{0}^{\frac{l+2}{2}}+\frac{2}{n+2} x_{0}^{\frac{n+2}{2}} s_{3}\right)\right]^{\frac{2}{l+2}}\right)\left[\frac{m+2}{2}\left(\frac{2}{m+2} y_{0}^{\frac{m+2}{2}}+\frac{2}{n+2} x_{0}^{\frac{n+2}{2}} s_{1}\right)\right]^{-\frac{m}{m+2}} \times \\
& \times\left[\frac{l+2}{2}\left(\frac{2}{k+2} z_{0}^{\frac{k+2}{2}}+\frac{2}{n+2} x_{0}^{\frac{n+2}{2}} s_{2}\right)\right]^{-\frac{k}{k+2}}\left[\frac{l+2}{2}\left(\frac{2}{l+2} t_{0}^{\frac{l+2}{2}}+\frac{2}{n+2} x_{0}^{\frac{n+2}{2}} s_{3}\right)\right]^{-\frac{l}{l+2}} d s_{1} d s_{2} d s_{3},
\end{aligned}
$$

where

$$
a=\frac{\frac{2}{m+2} y_{0}^{\frac{m+2}{2}}}{\frac{2}{n+2} x_{0}^{\frac{n+2}{2}}}, b=\frac{\frac{2}{k+2} z_{0}^{\frac{k+2}{2}}}{\frac{2}{n+2} x_{0}^{\frac{n+2}{2}}}, c=\frac{\frac{2}{l+2} t_{0}^{\frac{l+2}{2}}}{\frac{2}{n+2} x_{0}^{\frac{n+2}{2}}} .
$$

Mathematics series. № 4(104)/2021 
At $x_{0} \rightarrow 0$ from (35) we have

$$
\begin{aligned}
& \lim _{x_{0} \rightarrow 0} P_{1}\left(0, y, z, t ; x_{0}, y_{0}, z_{0}, t_{0}\right)=\sum_{l_{1}, l_{2}, l_{3}=0}^{\infty} \frac{(3-\alpha-\beta+\gamma+\delta)_{l_{1}+l_{2}+l_{3}}(1-\beta)_{l_{1}+l_{2}}(\gamma)_{l_{1}+l_{3}}(\delta)_{l_{2}+l_{3}}}{(2-2 \beta)_{l_{1}+l_{2}}(2 \gamma)_{l_{1}+l_{3}}(2 \delta)_{l_{2}+l_{3}} l_{1} ! l_{2} ! l_{3} !} \\
& \times F\left(\alpha-\beta-\gamma-\delta-1,1-\beta+l_{1}+l_{2} ; 2-2 \beta+l_{1}+l_{2} ; 1\right) \\
& \times F\left(\alpha+\beta+\gamma-\delta-3-l_{2}, \gamma+l_{1}+l_{3} ; 2 \gamma+l_{1}+l_{3} ; 1\right) \\
& \times F\left(\alpha+\beta-\gamma+\delta-3-l_{1}, \delta+l_{2}+l_{3} ; 2 \delta+l_{2}+l_{3} ; 1\right)
\end{aligned}
$$

Applying formulas (2) and (5) to (39), we determine

$$
\lim _{x_{0} \rightarrow 0} P_{1}\left(0, y, z, t ; x_{0}, y_{0}, z_{0}, t_{0}\right)=\frac{\Gamma(2-2 \beta) \Gamma(2 \gamma) \Gamma(2 \delta) \Gamma(2-\alpha)}{\Gamma(3-\alpha-\beta+\gamma+\delta) \Gamma(1-\beta) \Gamma(\gamma) \Gamma(\delta)}
$$

By virtue of (40), from (38) at $x_{0} \rightarrow 0$, we obtain

$$
\begin{aligned}
& \lim _{x_{0} \rightarrow 0} I_{1}\left(x_{0}, y_{0}, z_{0}, t_{0}\right)=k_{6}\left(\frac{8}{n+2}\right)^{\frac{2}{n+2}}\left(\frac{4}{m+2}\right)^{-2 \beta}\left(\frac{4}{k+2}\right)^{-2 \gamma}\left(\frac{4}{l+2}\right)^{-2 \delta} \times \\
& \times \frac{\Gamma(2-2 \beta) \Gamma(2 \gamma) \Gamma(2 \delta) \Gamma(2-\alpha)}{\Gamma(3-\alpha-\beta+\gamma+\delta) \Gamma(1-\beta) \Gamma(\gamma) \Gamma(\delta)} \tau_{1}\left(y_{0}, z_{0}, t_{0}\right) \int_{-\infty}^{\infty} \int_{-\infty}^{\infty} \int_{-\infty}^{\infty} \frac{d s_{1} d s_{2} d s_{3}}{\left(1+s_{1}^{2}+s_{2}^{2}+s_{3}^{2}\right)^{2-\alpha}} .
\end{aligned}
$$

To calculate the triple integral from (41), using formula (10), we get

$$
\int_{-\infty}^{\infty} \int_{-\infty}^{\infty} \int_{-\infty}^{\infty} \frac{d s_{1} d s_{2} d s_{3}}{\left(1+s_{1}^{2}+s_{2}^{2}+s_{3}^{2}\right)^{2-\alpha}}=8 \int_{0}^{\infty} \int_{0}^{\infty} \int_{0}^{\infty} \frac{d s_{1} d s_{2} d s_{3}}{\left(1+s_{1}^{2}+s_{2}^{2}+s_{3}^{2}\right)^{2-\alpha}}=\frac{\pi \sqrt{\pi} \Gamma\left(\frac{1}{2}-\alpha\right)}{\Gamma(2-\alpha)}
$$

Applying formula (1) in (42), as a result, we have

$$
\int_{-\infty}^{\infty} \int_{-\infty}^{\infty} \int_{-\infty}^{\infty} \frac{d s_{1} d s_{2} d s_{3}}{\left(1+s_{1}^{2}+s_{2}^{2}+s_{3}^{2}\right)^{2-\alpha}}=\frac{\pi^{2} \Gamma(2-2 \alpha)}{2^{-2 \alpha} \Gamma(2-\alpha)(1-2 \alpha) \Gamma(1-\alpha)}
$$

Substituting (43) into (41), we finally have

$$
\begin{aligned}
& \lim _{x_{0} \rightarrow 0} I_{1}\left(x_{0}, y_{0}, z_{0}, t_{0}\right)=4 \pi^{2} k_{6}\left(\frac{4}{n+2}\right)^{-2 \alpha}\left(\frac{4}{m+2}\right)^{-2 \beta}\left(\frac{4}{k+2}\right)^{-2 \gamma}\left(\frac{4}{l+2}\right)^{-2 \delta} \times \\
& \times \frac{\Gamma(2-2 \alpha) \Gamma(2-2 \beta) \Gamma(2 \gamma) \Gamma(2 \delta)}{\Gamma(3-\alpha-\beta+\gamma+\delta) \Gamma(1-\alpha) \Gamma(1-\beta) \Gamma(\gamma) \Gamma(\delta)} \tau_{1}\left(y_{0}, z_{0}, t_{0}\right)
\end{aligned}
$$

Taking into account (28), from (44), we obtain

$$
\lim _{x_{0} \rightarrow 0} I_{1}\left(x_{0}, y_{0}, z_{0}, t_{0}\right)=\tau_{1}\left(y_{0}, z_{0}, t_{0}\right) .
$$

It is easy to show that

$$
\lim _{x_{0} \rightarrow 0} I_{2}\left(x_{0}, y_{0}, z_{0}, t_{0}\right)=0, \lim _{x_{0} \rightarrow 0} I_{3}\left(x_{0}, y_{0}, z_{0}, t_{0}\right)=0, \lim _{x_{0} \rightarrow 0} I_{4}\left(x_{0}, y_{0}, z_{0}, t_{0}\right)=0 .
$$

Accordingly, $\lim _{x_{0} \rightarrow 0} u\left(x_{0}, y_{0}, z_{0}, t_{0}\right)=\tau_{1}\left(y_{0}, z_{0}, t_{0}\right)$, hence, function (29) satisfies condition (14) of the problem $\mathrm{ND}_{2}$. Similarly, can be convinced that function (29) also satisfies conditions (15), (16), and (17) of the problem $\mathrm{ND}_{2}$.

Let us show that if the given functions satisfy inequalities (19) - (22) for large enough values of the argument, then the solution (29) of the Problem $N D_{2}$ also satisfies condition (18). Indeed, let inequalities (19) - (22) are hold, in expressions (30) - (33) we make the following change of variables

$$
\begin{aligned}
& \xi_{1}=\frac{1}{R_{0}} \frac{2}{n+2} x^{\frac{n+2}{2}}, \eta_{1}=\frac{1}{R_{0}} \frac{2}{m+2} y^{\frac{m+2}{2}}, \zeta_{1}=\frac{1}{R_{0}} \frac{2}{k+2} z^{\frac{k+2}{2}}, \varsigma_{1}=\frac{1}{R_{0}} \frac{2}{l+2} t^{\frac{l+2}{2}}, \\
& \sigma_{1}=\frac{1}{R_{0}} \frac{2}{n+2} x_{0}^{\frac{n+2}{2}}, \sigma_{2}=\frac{1}{R_{0}} \frac{2}{m+2} y_{0}^{\frac{m+2}{2}}, \sigma_{3}=\frac{1}{R_{0}} \frac{2}{k+2} z_{0}^{\frac{k+2}{2}}, \sigma_{4}=\frac{1}{R_{0}} \frac{2}{l+2} t_{0}^{\frac{l+2}{2}},
\end{aligned}
$$


where

$$
R_{0}^{2}=\frac{4}{(n+2)^{2}} x_{0}{ }^{n+2}+\frac{4}{(m+2)^{2}} y_{0}{ }^{m+2}+\frac{4}{(k+2)^{2}} z_{0}{ }^{k+2}+\frac{4}{(l+2)^{2}} t_{0}^{l+2} .
$$

Then at $R_{0} \rightarrow \infty$ from (30)-(33) we obtain the following inequalities:

$$
\begin{aligned}
& \lim _{R_{0} \rightarrow \infty}\left|I_{1}\left(x_{0}, y_{0}, z_{0}, t_{0}\right)\right| \leq \frac{k_{6} c_{1}}{R_{0}^{2 \varepsilon_{1}}} 4^{\frac{2}{n+2}+\frac{2}{m+2}}\left(\sigma_{1}\right)^{\frac{2}{n+2}}\left(\sigma_{2}\right)^{\frac{2}{m+2}}\left(\frac{2}{n+2}\right)^{1-2 \alpha}\left(\frac{2}{m+2}\right)^{-2 \beta} \times \\
& \left(\frac{2}{k+2}\right)^{-2 \gamma}\left(\frac{2}{l+2}\right)^{-2 \delta} \int_{0}^{\infty} \int_{0}^{\infty} \int_{0}^{\infty} \frac{\eta_{1} \zeta_{1}{ }^{2 \gamma} \varsigma_{1}{ }^{2 \delta}}{\left(1+\eta_{1}{ }^{2}+\zeta_{1}{ }^{2}+\varsigma_{1}{ }^{2}\right)^{3-\alpha-\beta+\gamma+\delta}\left(\eta_{1}{ }^{2}+\zeta_{1}{ }^{2}+\varsigma_{1}{ }^{2}\right)^{\varepsilon_{1}}} d \eta_{1} d \zeta_{1} d \varsigma_{1}, \\
& \lim _{R_{0} \rightarrow \infty}\left|I_{2}\left(x_{0}, y_{0}, z_{0}, t_{0}\right)\right| \leq \frac{k_{6} c_{2}}{R_{0}^{2 \varepsilon_{2}}} 4^{\frac{2}{n+2}+\frac{2}{m+2}}\left(\sigma_{1}\right)^{\frac{2}{n+2}}\left(\sigma_{2}\right)^{\frac{2}{m+2}}\left(\frac{2}{n+2}\right)^{-2 \alpha}\left(\frac{2}{m+2}\right)^{1-2 \beta} \times \\
& \left(\frac{2}{k+2}\right)^{-2 \gamma}\left(\frac{2}{l+2}\right)^{-2 \delta} \int_{0}^{\infty} \int_{0}^{\infty} \int_{0}^{\infty} \frac{\xi_{1} \zeta_{1}{ }^{2 \gamma} \varsigma_{1}{ }^{2 \delta}}{\left(1+\xi_{1}{ }^{2}+\zeta_{1}{ }^{2}+\varsigma_{1}\right)^{3-\alpha-\beta+\gamma+\delta}\left(\xi_{1}{ }^{2}+\zeta_{1}{ }^{2}+\varsigma_{1}{ }^{2}\right)^{\varepsilon_{2}}} d \xi_{1} d \zeta_{1} d \varsigma_{1} \\
& \lim _{R_{0} \rightarrow \infty}\left|I_{3}\left(x_{0}, y_{0}, z_{0}, t_{0}\right)\right| \leq \frac{k_{6} c_{3}}{R_{0}^{\varepsilon_{3}}} 4^{\frac{2}{n+2}+\frac{2}{m+2}}\left(\sigma_{1}\right)^{\frac{2}{n+2}}\left(\sigma_{2}\right)^{\frac{2}{m+2}}\left(\frac{2}{n+2}\right)^{-2 \alpha}\left(\frac{2}{m+2}\right)^{-2 \beta} \times \\
& \left(\frac{2}{l+2}\right)^{-2 \delta} \int_{0}^{\infty} \int_{0}^{\infty} \int_{0}^{\infty} \frac{\xi_{1} \eta_{1} \varsigma_{1}{ }^{2 \delta}}{\left(1+\xi_{1}{ }^{2}+\eta_{1}{ }^{2}+\varsigma_{1}{ }^{2}\right)^{3-\alpha-\beta+\gamma+\delta}\left(\xi_{1}{ }^{2}+\eta_{1}{ }^{2}+\varsigma_{1}{ }^{2}\right)^{\frac{1-2 \gamma+\varepsilon_{3}}{2}}} d \xi_{1} d \eta_{1} d \varsigma_{1}, \\
& \lim _{R_{0} \rightarrow \infty}\left|I_{4}\left(x_{0}, y_{0}, z_{0}, t_{0}\right)\right| \leq \frac{k_{6} c_{4}}{R_{0}^{\varepsilon_{4}}} 4^{\frac{2}{n+2}+\frac{2}{m+2}}\left(\sigma_{1}\right)^{\frac{2}{n+2}}\left(\sigma_{2}\right)^{\frac{2}{m+2}}\left(\frac{2}{n+2}\right)^{-2 \alpha}\left(\frac{2}{m+2}\right)^{-2 \beta} \times \\
& \left(\frac{2}{k+2}\right)^{-2 \gamma} \int_{0}^{\infty} \int_{0}^{\infty} \int_{0}^{\infty} \frac{\xi_{1} \eta_{1} \zeta_{1}{ }^{2 \gamma}}{\left(1+\xi_{1}{ }^{2}+\eta_{1}{ }^{2}+\zeta_{1}{ }^{2}\right)^{3-\alpha-\beta+\gamma+\delta}\left(\xi_{1}{ }^{2}+\eta_{1}{ }^{2}+\zeta_{1}{ }^{2}\right)^{\frac{1-2 \delta+\varepsilon_{4}}{2}}} d \xi_{1} d \eta_{1} d \zeta_{1} .
\end{aligned}
$$

Let us show that the triple integrals in inequalities $(45)-(48)$ are bounded.

Considering the integrals from inequality (45) - (46), these integrals satisfy the identity.

$$
\begin{aligned}
& \int_{0}^{\infty} \int_{0}^{\infty} \int_{0}^{\infty} \frac{x y^{2 b} z^{2 c} d x d y d z}{\left(1+x^{2}+y^{2}+z^{2}\right)^{3-a-b+c+d}\left(x^{2}+y^{2}+z^{2}\right)^{\varepsilon}}= \\
= & \frac{1}{8} \frac{\Gamma\left(\frac{1}{2}+c\right) \Gamma\left(\frac{1}{2}+d\right) \Gamma(2+c+d-\varepsilon) \Gamma(1-a-b+\varepsilon)}{\Gamma(2+c+d) \Gamma(3-a-b+c+d)}, a+b-1<\varepsilon<2+c+d .
\end{aligned}
$$

Indeed, in integral (49), passing into spherical coordinates, we have

$$
\begin{aligned}
& \int_{0}^{\infty} \int_{0}^{\infty} \int_{0}^{\infty} \frac{x y^{2 b} z^{2 c} d x d y d z}{\left(1+x^{2}+y^{2}+z^{2}\right)^{3-a-b+c+d}\left(x^{2}+y^{2}+z^{2}\right)^{\varepsilon}}= \\
= & \int_{0}^{\frac{\pi}{2}} \sin ^{2 c} \varphi \cos \varphi d \varphi \int_{0}^{\frac{\pi}{2}} \sin ^{2+2 c} \theta \cos ^{2 d} \theta d \theta \int_{0}^{\infty} r^{3+2 c+2 d-2 \varepsilon}\left(1+r^{2}\right)^{a+b-c-d-3} d r .
\end{aligned}
$$

Using the values of integrals (11) and (12) in expression (50), we obtain the identity (49)

$$
\begin{aligned}
& \int_{0}^{\frac{\pi}{2}} \sin ^{2 c} \varphi \cos \varphi d \varphi \int_{0}^{\frac{\pi}{2}} \sin ^{2+2 c} \theta \cos ^{2 d} \theta d \theta \int_{0}^{\infty} r^{3+2 c+2 d-2 \varepsilon}\left(1+r^{2}\right)^{a+b-c-d-3} d r= \\
& =\frac{1}{8} \frac{\Gamma\left(\frac{1}{2}+c\right) \Gamma\left(\frac{1}{2}+d\right) \Gamma(2+c+d-\varepsilon) \Gamma(1-a-b+\varepsilon)}{\Gamma(2+c+d) \Gamma(3-a-b+c+d)}, a+b-1<\varepsilon<2+c+d .
\end{aligned}
$$

Mathematics series. № 4(104)/2021 
Thus, inequalities (45) - (46) by virtue of the value of integral (49) the inequalities follow

$$
\lim _{R_{0} \rightarrow \infty}\left|I_{1}\left(x_{0}, y_{0}, z_{0}, t_{0}\right)\right| \leq \frac{k_{6} \overline{c_{1}}}{R_{0}^{2 \varepsilon_{1}}}, \lim _{R_{0} \rightarrow \infty}\left|I_{2}\left(x_{0}, y_{0}, z_{0}, t_{0}\right)\right| \leq \frac{k_{6} \overline{c_{2}}}{R_{0}^{2 \varepsilon_{2}}}
$$

where $\overline{c_{1}}, \overline{c_{2}}$ are constants.

Let us show that the integrals in $(47)-(48)$ are bounded. For inequalities $(47)-(48)$, the identity is true

$$
\begin{aligned}
& \int_{0}^{\infty} \int_{0}^{\infty} \int_{0}^{\infty} \frac{x y t^{2 d}}{\left(1+x^{2}+y^{2}+t^{2}\right)^{3-a-b+c+d}\left(x^{2}+y^{2}+t^{2}\right)^{\frac{1-2 c+\varepsilon}{2}}} d x d y d t= \\
& =\frac{1}{8} \frac{\Gamma\left(\frac{1}{2}+d\right)}{\Gamma\left(\frac{5}{2}+d\right)} \frac{\Gamma\left(2+c+d-\frac{\varepsilon}{2}\right) \Gamma\left(1-a-b+\frac{\varepsilon}{2}\right)}{\Gamma(3-a-b+c+d)}, \quad 2 a+2 b-2<\varepsilon<4+2 c+2 d .
\end{aligned}
$$

Passing into spherical coordinates in (52), we obtain

$$
\begin{aligned}
& \int_{0}^{\infty} \int_{0}^{\infty} \int_{0}^{\infty} \frac{x y t^{2 d}}{\left(1+x^{2}+y^{2}+t^{2}\right)^{3-a-b+c+d}\left(x^{2}+y^{2}+t^{2}\right)^{\frac{1-2 c+\varepsilon}{2}}} d x d y d t= \\
& =\int_{0}^{\frac{\pi}{2}} \sin \varphi \cos \varphi d \varphi \int_{0}^{\frac{\pi}{2}} \sin ^{3} \theta r \cos ^{2 d} \theta d \theta \int_{0}^{\infty}\left(1+r^{2}\right)^{a+b-c-d-3} r^{3+2 c+2 d-\varepsilon} d r .
\end{aligned}
$$

Using formulas (11) and (12) to the right-hand side of (53), we define

$$
\begin{aligned}
& \int_{0}^{\frac{\pi}{2}} \sin \varphi \cos \varphi d \varphi \int_{0}^{\frac{\pi}{2}} \sin ^{3} \theta r \cos ^{2 d} \theta d \theta \int_{0}^{\infty}\left(1+r^{2}\right)^{a+b-c-d-3} r^{3+2 c+2 d-\varepsilon} d= \\
& =\frac{1}{8} \frac{\Gamma\left(\frac{1}{2}+d\right)}{\Gamma\left(\frac{5}{2}+d\right)} \frac{\Gamma\left(2+c+d-\frac{\varepsilon}{2}\right) \Gamma\left(1-a-b+\frac{\varepsilon}{2}\right)}{\Gamma(3-a-b+c+d)}, 2 a+2 b-2<\varepsilon<4+2 c+2 d .
\end{aligned}
$$

Thus, we have shown that the integrals in inequalities (47) - (48) are bounded; the integrals satisfy the inequalities

$$
\lim _{R_{0} \rightarrow \infty}\left|I_{3}\left(x_{0}, y_{0}, z_{0}, t_{0}\right)\right| \leq \frac{k_{6} \overline{c_{3}}}{R_{0}{ }^{\varepsilon_{3}}}, \lim _{R_{0} \rightarrow \infty}\left|I_{4}\left(x_{0}, y_{0}, z_{0}, t_{0}\right)\right| \leq \frac{k_{6} \overline{c_{4}}}{R_{0}{ }^{\varepsilon_{4}}},
$$

where $\overline{c_{3}}, \overline{c_{4}}$ are constants. Inequalities (51) and (54) show that solution (27) at $R_{0} \rightarrow \infty$ tends to zero. Thereby, condition (18) of Problem $N D_{2}$ is satisfied. In this connection, solution (27) of Problem $N D_{2}$ satisfies all conditions of Problem $\mathrm{ND}_{2}$.

\section{Conclusions}

We have proved the following theorem.

Theorem 2. Let conditions (19) - (22) be satisfied, then a regular solution to problem $N D_{2}(13),(14)-(18)$ exists and is expressed by formula (27).

In four-dimensional space in an infinite domain for the degenerate elliptic Gellerstedt equation, the problem $\mathrm{ND}_{2}$ with two Neumann boundary conditions and with two Dirichlet conditions is solved. The solution is written explicitly. The uniqueness and existence of a solution to the equation are proved.

\section{Acknowledgments}

This research was funded by the Grant of Abai Kazakh National Pedagogical University. 


\section{References}

1 Коган М.Н. О магнитогидродинамических течениях смешанного типа / М.Н. Коган // Прикладная математика и механика. - 1961. - 25(1). - С. 132-137.

2 Франкль Ф.И. Избранные труды по газовой динамике / Ф.И. Франкль. - М.: Наука, $1973 .-712$ с.

3 Бицадзе А.В. Некоторые классы уравнений в частных производных / А.В. Бицадзе. - М.: Наука, 1981. - 448 c.

4 Смирнов М.М. Вырождающиеся эллиптические и гиперболические уравнения / М.М. Смирнов. М.: Наука, 1966. - 292 с.

5 Altin A. Solutions of type for a class of singular equations / A. Altin // International Journal of Mathematical Science. - 1982. - 5(3). - P. 613-619.

6 Gilbert R. Function Theoretic Methods in Partial Differential Equations / R. Gilbert. - New York, London: Academic Press, 1969.

7 Смирнов М.М. Уравнения смешанного типа / М.М. Смирнов. - М.: Высш. шк., 1985. - 304 с.

8 Agmon S. The fundamental solution and Tricomi's problem for a class of equations of mixed type / S. Agmon. - Amsterdam: Proc. Internat. Cong. Math. - 1954. - II.

9 Bers L. Mathematical Aspects of Subsonic and Transonic Gas Dynamics / L. Bers. - New York: Wiley, 1958.

10 Luke Y.L. The Special Functions and Their Approximations / Y.L. Luke. - New York, London: Acad. Press., 1969. - V. 1.

11 Mathai A.M. Generalized Hypergeometric Functions with Applications in Statistics and Physical Sciences / A.M .Mathai, R.K. Saxena // Lecture Notes in Mathematics. - 1973.

12 Niukkanen A.W. Generalised hypergeometric series $\mathrm{NF}(\mathrm{x} 1, \ldots$, . xN) arising in physical and quantum chemical applications / A.W. Niukkanen // Journal of Physics A: Mathematical and General. - 1983. 16. - P. 1813-1825.

13 Rassias J.M. A maximum principle in $\left[R^{n+1} /\right.$ J.M. Rassias // Journal of Mathematical Analysis and Applications. - 1982. - 85. - No.1. - P. 106-113.

14 Sneddon I.N. Special Functions of Mathematical Physics and Chemistry / I.N. Sneddon. - London and New York: Longman, 1980. - Third ed.

15 Fryant A.J. Growth and complete sequences of generalized bi-axially symmetric potentials / A.J. Fryant // Journal of Differential Equations. - 1979. - 31(2). - P. 155-164.

16 Hasanov A. Fundamental solutions for a class of three-dimensional elliptic equations with singular coefficients / A. Hasanov, E.T. Karimov // Applied Mathematics Letters. - 2009. - 22. — P. 1828-1832.

17 Karimov E.T. On a boundary problem with Neumann's condition for 3D singular elliptic equations / E.T. Karimov // Applied Mathematics Letters. - 2010. - 23. - P. 517-522.

18 Kitagawa T. On the numerical stability of the method of fundamental solution applied to the Dirichlet problem / T. Kitagawa // Japan Journal of Applied Mathematics. - 1988. - 5. - P. 123-133.

19 Salakhitdinov M.S. A solution of the Neumann-Dirichlet boundary-value problem for generalized biaxially symmetric Helmholtz equation / M.S. Salakhitdinov, A. Hasanov // Complex Variables and Elliptic Equations. - 2008. - 53(4). - P.355-364.

20 Hasanov A. Fundamental solutions for a class of four-dimensional degenerate elliptic equation / A. Hasanov, A.S. Berdyshev, A. Ryskan // Complex Variables and Elliptic Equations. - 2020. - 65(4). - P. $632-647$.

21 Berdyshev A.S. The Neumann and Dirichlet problems for one four-dimensional degenerate elliptic equation / A.S. Berdyshev, A. Ryskan // Lobachevskii Journal of Mathematics. - 2020. - 41(6). P. 1051-1066.

22 Бердышев А.С. Краевая задача для одного класса четырехмерных вырождающихся эллиптических уравнений / А.С. Бердышев, А.Х. Хасанов, А. Рыскан // Итоги науки и техники. Сер. Современная математика и ее приложения. Тематические обзоры. - 2021. - Т. 194. - С. 55-70.

23 Berdyshev A.S. Solution of the Neumann problem for one fourdimensional elliptic equation / A.S. Berdyshev, A Hasanov, A.R. Ryskan // Eurasian mathematical journal. - 2020. - 11(2). — P. 93-97 
24 Бейтмен Г. Высшие трансцендентные функции. Гипергеометрические функции. Функции Лежандра / Г. Бейтмен, А. Эрдейи. - М.: Наука, 1973. - 296 с.

25 Appell P. Fonctions Hypergeometriques et Hyperspheriques; Polynomes d'Hermite / P. Appell, J. Kampe de Feriet. - Paris: Gauthier-Villars, 1926.

26 Erdelyi A. Higher transcendental functions / A. Erdelyi, W. Magnus, F. Oberhettinger, F.G. Tricomi. New York-Toronto-London: McGraw-Hill Book Company, Inc. - 1953. - Vol. I.

27 Hasanov A. Some decomposition formulas associated with the Lauricella function and other multiple hypergeometric functions / A. Hasanov, H.M. Srivastava // Applied Mathematics Letters. - 2006. 19(2). - Р. 113-121.

28 Градштейн И.С. Таблицы интегралов, сумм, рядов и произведений. - 4-е изд. / И.С. Градштейн, И.М. Рыжик. - М.: Физматгиз, 1963. - 1100 с.

\author{
А.С. Бердышев ${ }^{1,2}$, А.Р. Рысқан ${ }^{1,2}$ \\ ${ }^{1}$ Абай атындавы Қазақ, ұлттық, педагогикалық, университеті, Алматы, Қазақстан; \\ ${ }^{2}$ Ақпараттық, және есептеуіш технологиялар институты, Алматы, Қазақстан
}

\title{
Төрт өлшемді Геллерстедт теңдеуі үшін шеттік есеп
}

\begin{abstract}
Мақалада төрт айнымалы Геллерстедт теңдеу үшін Нейман және Дирихле шарттары бар шеттік есептің шешілуі зерттелген. Есеп шешімінің жалғыздығын дәлелдеу үшін энергия интегралы әдісі қолданылған. Сонымен қатар, шешімінің бар болуына гипергеометриялық функцияларды дифференциациялау, автотрансформациялау және жіктеу формулалары пайдаланылған. Шешім айқын түрде алынған және Лауричелла гипергеометриялық функцияларымен өрнектелген.
\end{abstract}

Kiлm сөздер: Геллерстедт теңдеуі, аралас шарттары бар шеттік есеп, фундаментальді шешім, Лауричелла гипергеометриялық функциясы.

\section{А.С. Бердышев ${ }^{1,2}$, А.Р. Рыскан ${ }^{1,2}$}

\footnotetext{
${ }^{1}$ Казахский национальный педагогический университет имени Абая, Алматы, Казахстан;

${ }^{2}$ Институт информационных и вычислительных технологий МОН РК, Алматы, Казахстан
}

\section{Краевая задача для четырехмерного уравнения Геллерстедта}

В статье исследована разрешимость задачи с краевыми условиями Неймана и Дирихле для уравнения Геллерстедта от четырех переменных. В ходе доказательства единственности решения задачи применен метод интеграла энергии, кроме того, существовании решения в задачи использованы формулы дифференцирования, автотрансформации, разложения гипергеометрических функций. Решение получено в явном виде и выражено гипергеометрическими функциями Лауричеллы.

Ключевые слова: уравнение Геллерстедта, краевая задача со смешанными условиями, фундаментальное решение, гипергеометрическая функция Лауричеллы.

\section{References}

1 Kogan, M.N. (1961). O magnitogidrodinamicheskikh techeniiakh smeshannogo tipa [Mixed type magnetohydrodynamic flows]. Prikladnaia matematika i mekhanika, 25(1), 132-137 [in Russian].

2 Frankl, F.I. (1973). Izbrannye trudy po gazovoi dinamike [Selected Works on Gas Dynamics]. Moscow: Nauka [in Russian]. 
3 Bitsadze, A.V. (1981). Nekotorye klassy uravnenii v chastnykh proizvodnykh /Some Classes of Partial Differential Equations]. Moscow: Nauka [in Russian].

4 Smirnov, M.M. (1966). Vyrozhdaiushchiesia ellipticheskie i giperbolicheskie uravneniia [Degenerate elliptic and hyperbolic equations]. Moscow: Nauka [in Russian].

5 Altin, A. (1982). Solutions of type for a class of singular equations. International Journal of Mathematical Science, 5(3), 613-619.

6 Gilbert, R. (1969). Function Theoretic Methods in Partial Differential Equations. New York-London: Academic Press.

7 Smirnov, M.M. (1985). Uravneniia smeshannogo tipa [Mixed type equations]. Moscow: Vysshaia shkola [in Russian].

8 Agmon, S. (1954). The fundamental solution and Tricomi's problem for a class of equations of mixed type. Amsterdam: Proc. Internat. Cong. Math.

9 Bers, L. (1958). Mathematical Aspects of Subsonic and Transonic Gas Dynamics. New York: Wiley.

10 Luke, Y.L. (1969). The Special Functions and Their Approximations. New York-London: Academic Press.

11 Mathai, A.M., \& Saxena, R.K. (1973). Generalized Hypergeometric Functions with Applications in Statistics and Physical Sciences. Lecture Notes in Mathematics.

12 Niukkanen, A.W. (1983). Generalised hypergeometric series $\mathrm{NF}(\mathrm{x} 1, \ldots$. . , xN) arising in physical and quantum chemical applications Journal of Physics A: Mathematical and General, 16, 1813-1825.

13 Rassias, J.M. (1982). A maximum principle in $\left[R^{n+1}\right.$. Journal of Mathematical Analysis and Applications, 85(1), 106-113.

14 Sneddon, I.N. (198). Special Functions of Mathematical Physics and Chemistry. London-New York: Longman. Third ed.

15 Fryant, A.J. (1979). Growth and complete sequences of generalized bi-axially symmetric potentials. Journal of Differential Equations, 31(2), 155-164.

16 Hasanov, A., \& Karimov, E.T. (2009). Fundamental solutions for a class of three-dimensional elliptic equations with singular coefficients. Applied Mathematics Letters, 22, 1828-1832.

17 Karimov, E.T. (2010). On a boundary problem with Neumann's condition for 3D singular elliptic equations. Applied Mathematics Letters, 23, 517-522.

18 Kitagawa, T. (1988). On the numerical stability of the method of fundamental solution applied to the Dirichlet problem. Japan Journal of Applied Mathematics, 5, 123-133.

19 Salakhitdinov, M.S. \& Hasanov, A. (2008). A solution of the Neumann-Dirichlet boundary-value problem for generalized bi-axially symmetric Helmholtz equation. Complex Variables and Elliptic Equations, 53(4), $355-364$.

20 Hasanov, A., Berdyshev, A.S., \& Ryskan, A. (2020). Fundamental solutions for a class of four-dimensional degenerate elliptic equation. Complex Variables and Elliptic Equations, 65(4), 632-647.

21 Berdyshev, A.S., \& Ryskan, A. (2020). The Neumann and Dirichlet problems for one four-dimensional degenerate elliptic equation. Lobachevskii Journal of Mathematics, 41(6), 1051-1066.

22 Berdyshev, A.S., Hasanov, A.H., \& Ryskan, A. (2021). Kraevaia zadacha dlia odnogo klassa chetyrekhmernykh vyrozhdaiushchikhsia ellipticheskikh uravnenii [A boundary value problem for a class of fourdimensional degenerate elliptic equations]. Itogi nauki $i$ tekhniki. Seriia Sovremennaia matematika $i$ ee prilozheniia. Tematicheskie obzory - Results of Science and Technology. Series Contemporary Mathematics and Its Applications. Thematic reviews, 194, 55-70 [in Russian].

23 Berdyshev, A.S., Hasanov, A.H., \& Ryskan, A. (2020). Solution of the Neumann problem for one fourdimensional elliptic equation. Eurasian mathematical journal, 11(2), 93-97.

24 Bateman, G. \& Erdelyi, A. (1973). Vysshiie transtsendentnye funktsii. Gipergeometricheskie funktsii. Funktsii Lezhandra. [Higher transcendental functions. Hypergeometric functions. Legendre functions Legendre functions.] Moscow: Nauka [in Russian].

25 Appell, P., \& Kampe de Feriet, J. (1926). Hypergeometriques et Hyperspheriques; Polynomes d'Hermite. Paris: Gauthier-Villars.

26 Erdelyi, A., Magnus, W., Oberhettinger, F., \& Tricomi, F.G. (1953). Higher transcendental functions. New York-Toronto-London: McGraw-Hill Book Company, Inc. 
27 Hasanov, A., \& Srivastava, H.M. (2006). Some decomposition formulas associated with the Lauricella function and other multiple hypergeometric functions. Applied Mathematics Letters, 19(2), $113-121$.

28 Gradshtein, I.S., \& Ryzhik, I.M. (1963). Tablitsy integralov, summ, riadov i proizvedenii [Tables of integrals, sums, series and products]. 4th ed. Moscow: Fizmatgiz [in Russian]. 